\title{
Plastische Rekonstruktion der Mamma - Eigengewebe
}

\author{
Justus P. Beier, Andreas Arkudas, Marweh Schmitz, Raymund E. Horch
}

\section{Übersicht}

\section{Einleitung}

Indikation zur autologen

Brustrekonstruktion mit

freien mikrochirurgischen

Lappentransplantationen

Freie muskelsparende ventrale
Verfahren (TRAM, DIEP, SIEA)
Freier transverser myokutaner
Gracilis-Lappen (TMG)
Lappen aus anderen
Spenderregionen

393

\section{Einleitung}

Die autologe Brustrekonstruktion wurde erstmalig 1979 von Holmström in Form des freien, an den inferioren epigastrischen Gefäßen frei transplantierten transversen Rectus-abdomins-myokutan-Lappens beschrieben [1]. Eine Pionierleistung, die oft nicht zu Recht gewürdigt wird, da in der Vielzahl der historischen Darstellungen diese Leistung Hartrampf zugeschrieben wird, der 1984 eine autologe Brustrekonstruktion in Form der an den superioren epigastrischen Gefäßen gestielten transversen myokutanen Rectusabdominis-Lappenplastik, dem sog. gestielten TRAM-Lappen durchführte und publizierte [2].

In den vergangenen 30 Jahren haben sich die verfügbaren Techniken und beschriebenen Hebestellen zur Gewinnung von Eigengewebstransplantaten für die Brustrekonstruktion radikal weiterentwickelt. Nicht zuletzt aufgrund der insgesamt zunehmenden mikrochirurgischen Expertise stellt die autologe Brustrekonstruktion mittels freier mikrochirurgischer Lappenplastiken daher heutzutage in entsprechenden Zentren ein Routineverfahren dar, dessen Überlegenheit sowohl hinsichtlich der kosmetischen Ergebnisse als auch bez. der Komplikationsraten und Sicherheit gegenüber den früheren Verfahren der gestielten Lappenplastiken in zahlreichen Studien belegt werden konnte.
Freie Lappenplastiken sind den gestielten Verfahren nachweislich überlegen.

In vorliegendem Übersichtsartikel wird die leitliniengerechte und patientinnenorientierte Indikationsstellung für die autologe mikrochirurgische Brustrekonstruktion erläutert, das praktische Vorgehen bei der interdisziplinären Planung im Brustzentrum dargestellt sowie nachfolgend eine Übersicht über die gängigsten Lappenplastiken und deren technische Durchführung gegeben.

\section{Indikation zur autologen Brustrekonstruktion mit freien mikrochirurgischen Lappentransplantationen}

Trotz der zunehmenden Anzahl brusterhaltend therapierter Mammakarzinome stellt die verbleibende Zahl der Patientinnen, welche eine Ablatio mammae erhalten, eine große Herausforderung für die rekonstruktive Chirurgie dar. Die Planung der Brustrekonstruktion sollte im Rahmen einer interdisziplinären Brustkrebskonferenz erfolgen und bereits bei der Indikation zur primären operativen Therapie der Brust berücksichtigt werden [3]. Die alloplastische Rekonstruktion mit Silikongelimplantaten stellt hierbei auch heute noch ein geeignetes und weit verbreitetes Verfahren zur Brustrekonstruktion dar. Dieses Verfahren geht mit spezi- 
fischen Vor- und Nachteilen einher, welche Gegenstand des entsprechenden Artikels in derselben Ausgabe dieser Fachzeitschrift (Rauh et al., S. 409) sind und daher hier nur kurz gestreift werden sollen.

Nachteile alloplastischer Verfahren. Während die geringe perioperative Komplikationsrate der Prothesenrekonstruktion auf der Hand liegt, sind die mittel- und langfristig möglichen Komplikationen schwieriger einzuordnen. Hierzu gehören in erster Linie mit dem implantierten Fremdkörper einhergehende Probleme, d.h. Protheseninfekte und -dislokationen sowie die letztlich nach einer gewissen Zeit mit hoher Wahrscheinlichkeit eintretende Kapselkontraktur. Letztere kann heutzutage relativ valide abgeschätzt werden anhand der größten Längsschnittstudie zu dieser Frage, welche durch die beiden amerikanischen Prothesenhersteller durchgeführt wurde als Voraussetzung für die 2006 erfolgte Beendigung des US-Prothesen-Moratoriums von 1992.

In den sog. „Adjunct Studies“ der beiden zu dieser Zeit in den USA von der FDA zugelassenen Silikongelprothesenhersteller waren 44799 Patientinnen (Fa. Allergan) bzw. 57828 (Fa. Mentor) eingeschlossen worden. Bereits nach 5 Jahren betrug in diesen zahlenmäßig größten prospektiven Studien die Wahrscheinlichkeit für das Eintreten einer revisionsbedürftigen Kapselkontraktur bei unbestrahlten Patientinnen 16\% [4]. Nach vorausgegangener Radiatio liegt diese signifikant höher und erreicht hierbei Werte, die letztlich dazu geführt haben, dass in der aktuell gültigen S3-Leitlinie zur Behandlung des Mammakarzinoms keine Silikongelprothesenimplantation bei bestrahlten Patientinnen mehr empfohlen wird: „Nach vorausgegangener Bestrahlung ist der Brustwiederaufbau mit körpereigenem Gewebe der Anwendung von Expandern oder Prothesen vorzuziehen (...)“ [5].

Nach Bestrahlung sollte keine Prothesenrekonstruktion der Brust erfolgen.

Beratung zur Brustrekonstruktion. Patientinnen, welche im Rahmen ihrer Beratung über die Notwendigkeit einer Ablatio mammae informiert werden, sollten grundsätzlich ausführlich über o.g. Fakten bez. der Prothesenrekonstruktion sowie über die verfügbaren Alternativen beraten werden. Die Empfehlung zur vollständigen und umfassenden Beratung und Aufklärung über alle möglichen Verfahren der Brustrekonstruktion, einschließlich der mikrochirurgischen autologen Brustrekonstruktion, ist daher auch in die aktuell gültige S3-Leitlinie aufgenommen worden: „Bei der prä- operativen Beratung sollten auch die Möglichkeiten zur Sofortrekonstruktion (Expander, gestielte und freie Lappenplastiken) umfassend diskutiert werden." Außerdem soll ,jede Patientin, bei der eine Mastektomie durchgeführt werden soll, (...) über die Möglichkeit einer sofortigen oder späteren Brustrekonstruktion bzw. den Verzicht auf rekonstruktive Maßnahmen aufgeklärt werden (...)“ [5].

Zeitliche Planung. Hinsichtlich des idealen Zeitpunktes der autologen Brustrekonstruktion bestehen je nach Befundkonstellation unterschiedliche Ansichten unter den rekonstruktiv tätigen Operateuren bez. folgender Fragen:

- Ist ein Eigengewebsaufbau vor oder nach einer noch geplanten Bestrahlung durchzuführen?

- Sollte eine primäre, d.h. im Rahmen der Mastektomie erfolgende Brustrekonstruktion angeboten werden?

Hinsichtlich der Bestrahlung besteht heutzutage größtenteils Einigkeit darüber, dass es vorteilhafter ist, wenn diese bereits vor dem Rekonstruktionseingriff erfolgt ist, da die durch die Bestrahlung hervorgerufenen, u.U. signifikanten Veränderungen im Lappen (Form, Konsistenz) nicht durch das bislang nicht belegte höhere Risiko eines Anschlusses an zuvor in der Nähe des Strahlenfelds gelegene Gefäße aufgewogen werden.

Obgleich einige Autoren die bei moderner dreidimensionaler Strahlenfeldplanung vorgesehene Dosis und damit verbundene strahlentherapieassoziierte Veränderung des Lappens als sehr gering einschätzen, ist die Mehrheit der rekonstruktiven Chirurgen dazu übergegangen, eine Eigengewebsrekonstruktion erst nach definitivem Abschluss der Strahlentherapie vorzunehmen $[6,7]$.

\section{Tipp für die Praxis}

Daten für ein genaues Mindestzeitfenster, welches nach Radiatio und vor Eigengewebsaufbau eingehalten werden sollte, existieren bis dato nicht; in der Praxis hat sich ein Mindestabstand von 3 Monaten nach Abschluss der Strahlentherapie bewährt.

Primäre Brustrekonstruktion? Schwieriger zu beantworten ist die Frage, ob und wenn ja, in welchen Fällen eine primäre Brustrekonstruktion indiziert ist, und wenn nicht, wann frühestens die sekundäre Rekonstruktion erfolgen sollte. Zwar gibt es einzelne Studien, die von einer höheren Zufriedenheitsrate und v.a. besserer psychischer Verarbeitung der Tumorerkrankung 
bei Patientinnen mit Primärrekonstruktion berichten. Diesem Vorgehen stehen allerdings folgende Probleme entgegen:

- rechtzeitige Erkennung eines frühzeitigen Lokalrezidivs

- die zum Mastektomie-/Rekonstruktionszeitpunkt noch nicht sicher absehbare Notwendigkeit einer adjuvanten Bestrahlung

- das Problem, im Falle eines frühen Lokalrezidivs den bereits transplantierten Lappen mitresezieren und verwerfen zu müssen - mit dann deutlich erschwerten Bedingungen für eine erneute Eigengewebsrekonstruktion

Sekundäre Rekonstruktion. Während in einigen Zentren eine Primärrekonstruktion bei allen Patientinnen, die grundsätzlich für einen Eigengewebsaufbau infrage kommen angeboten wird, hat sich in anderen - so auch dem Universitäts-Brustzentrum Franken des Universitätsklinikums Erlangen - ein anderer Standard bewährt: Patientinnen mit invasiven Karzinomen bekommen eine sekundäre Brustrekonstruktion nach einem Jahr unauffälliger Nachsorge angeboten, während bei Patientinnen mit DCIS (und natürlich bei prophylaktischer Mastektomie) eine Primärrekonstruktion mit Eigengewebe durchgeführt wird.

Sekundäre Brustrekonstruktion nach invasiven Karzinomen, primäre Rekonstruktion nach DCIS und prophylaktischer Mastektomie.

Eigengewebsaufbau. Zur Frage der Prothesenrekonstruktion nach vorausgegangener Bestrahlung ist die Studienlage und die daraus resultierende Empfehlung zum Eigengewebsaufbau eindeutig. Hingegen wird die grundsätzliche Entscheidung bez. eines Eigengewebsaufbaus bei nicht bestrahlten Patientinnen in erster Linie durch mehrere Parameter bestimmt, auf welche im Folgenden genauer eingegangen werden soll.

\section{Wünsche der Patientinnen}

Während bei der Beratung der Patientin sowohl das individuelle Risikoprofil als auch die jeweilige Gewebeverfügbarkeit eine wichtige Rolle aus Sicht des rekonstruktiven Chirurgen darstellen, ist der Wunsch der Patientin bez. des Eigengewebsaufbaus der wichtigste Faktor bei der Entscheidungsfindung.

Angst vor Komplikationen. Hier spielen persönliche Gründe wie Angst vor fremdkörperassoziierten Nebenwirkungen oder Folgeoperationen, die für einen
Eigengewebsaufbau sprächen, ebenso eine entscheidende Rolle wie eventuell bereits gemachte Vorerfahrungen mit komplikationsbehafteten Verläufen nach alloplastischer oder autologer Brustrekonstruktion.

Kosmetisches Ergebnis. Eine wichtige Rolle spielen auch die persönlichen Wünsche der Patientin an das rekonstruktive Ergebnis, d.h. wie wichtig z.B. die Erzielung einer natürlichen Brustform ist und darüber hinaus, ob zu diesem Zeitpunkt eine Akzeptanz bez. des mit dem Eigengewebsaufbau verbundenen höheren operativen Aufwands und längeren stationären Aufenthalts gegeben ist. Des Weiteren ist der Wunsch der Patientin nach der Vermeidung von Narben an anderen Körperstellen (Hebestelle für Eigengewebstransplantate) gelegentlich ausschlaggebend.

Qualität der Beratung. Nicht zuletzt wird der Wunsch der Patientin jedoch maßgeblich von dem Umfang und der Qualität der ihr vorliegenden Informationen über die verschiedenen Rekonstruktionsverfahren bestimmt, sodass dem beratenden und aufklärenden rekonstruktiven Operateur, unabhängig von seiner Fachrichtung, hier eine besondere Verantwortung zukommt.

\section{Angestrebtes rekonstruktives Ergebnis}

Während bei einer jungen Patientin mit einseitigem Befall der Brust und verhältnismäßig straffem Hautmantel und geringer Ptosis mammae der Gegenseite ein sehr gutes kosmetisches Ergebnis durch Rekonstruktion der abladierten Brust mittels Prothesenimplantation erzielt werden kann, wird dies mit zunehmendem Alter, bzw. Größe/Schwere und damit Ptose der gegenseitigen Brust immer schwieriger. Selbst bei geplanter Mastopexie/Mammareduktionsplastik der Gegenseite ist daher in Abhängigkeit von der vorliegenden Ptose der Gegenseite und Haut-/ Weichteilbeschaffenheit der betroffenen Seite eine natürliche Brustform häufig nur durch eine Eigengewebsrekonstruktion zu erzielen. Mit diesen Verfahren kann neben dem erforderlichen Volumen auch ein

Parameter für die Indikationsstellung zur Eigengewebsrekonstruktion der Brust

- Wunsch der Patientin

- angestrebtes rekonstruktives Ergebnis

- lokale Gewebeverfügbarkeit an potenziellen Entnahmestellen

- individuelles Risikoprofil der Patientin 
signifikanter Teil des Haut-/Weichteilmantels der neuen Brust mit rekonstruiert werden und damit insbesondere im unteren Brustpol eine natürliche Form erzielt werden.

Mit zunehmendem Alter der Patientin wird eine alloplastische Rekonstruktion schwieriger.

Auch bei Patientinnen mit (z.B. aufgrund von Voroperationen) stark kompromittierten Haut-/Weichteilverhältnissen im Bereich der rekonstruierten Brust bietet der Eigengewebsaufbau den grundsätzlichen Vorteil, neben dem Volumen einen Ersatz durch den (nicht vorgeschädigten) Hautmantel zu gewährleisten, was in diesen Fällen einen signifikanten Vorteil bietet sowohl gegenüber der alleinigen Prothesenimplantation als auch der Implantation eines Expanders mit nachfolgendem Wechsel auf eine Silikongelprothese.

\section{Lokale Gewebeverfügbarkeit an potenziellen Entnahmestellen}

In den ersten Jahren nach Erstbeschreibung der autologen Brustrekonstruktion standen ausschließlich die beiden folgenden Entnahmestellen für die Rekonstruktion zur Verfügung:

- Unterbauch im Sinne eines zunächst kranial gestielten, später und bis heute fast ausschließlich mikrochirurgisch frei zu transplantierenden TRAM-Lappens (s.u.)

- Rücken im Sinne eines gestielten Latissimus-dorsiTransfers

Bei Letzterem ist in der Regel allerdings auch heute noch eine zusätzliche Prothesenimplantation erforderlich, um ein ausreichendes zu rekonstruierendes Brustvolumen zu erzielen, sodass dieses Verfahren eine Ausnahmestellung im Rahmen des rekonstruktiven Algorithmus hat und nur noch speziellen Fällen vorbehalten sein sollte (s.u.).

\section{Tipp für die Praxis}

Falls bei der Patientin ausreichendes Gewebe im Bereich des Unterbauchs, d. h. zwischen Bauchnabel (kranial) und Symphyse (kaudal), vorhanden ist, stellt dies nach einhelliger Meinung der rekonstruktiv tätigen Brustchirurgen die Hebestelle der ersten Wahl dar.
Aufgrund von Voroperationen oder unzureichender abdomineller Gewebeverfügbarkeit kommt die Entnahme von Unterbauchgewebe bei manchen Patientinnen jedoch nicht infrage. Daher wurden im Verlauf der vergangenen 2 Jahrzehnte alternative Entnahmestellen untersucht und freie Lappenplastiken aus diesen Regionen beschrieben, die heute zur klinischen Routine in entsprechenden plastisch-chirurgischen Zentren gehören. Hierzu gehören in erster Linie die Lappen von der Innenseite der Oberschenkel (sog. TMG-Lappen; s.u.), der oberen (sog. S-GAP-Lappen) und der unteren Glutealregion (sog. I-GAP- und FCILappen). Wegen der genannten alternativen Entnahmestellen ist daher in sehr seltenen Ausnahmefällen eine Diskrepanz zwischen Gewebeverfügbarkeit aus einer der o.g. Regionen und zu erzielendem Rekonstruktionsvolumen gegeben.

Der mikrochirurgische Eigengewebsaufbau ist nur in seltenen Ausnahmefällen durch unzureichende Gewebeverfügbarkeit an potenziellen Entnahmestellen limitiert.

\section{Tipp für die Praxis}

Im klinischen Alltag korreliert das angestrebte Rekonstruktionsvolumen i.d. R. sehr stark mit der vorhandenen Gewebeverfügbarkeit an Bauch und/ oder Oberschenkelinnenseite bzw. Gesäß.

\section{Individuelles Risikoprofil der Patientin}

Die Verfahren der mikrochirurgischen Eigengewebsrekonstruktion gehen in der Regel mit deutlich längeren Operationszeiten - verglichen mit einer Prothesenimplantation - einher, sodass zumindest bei stark reduziertem Allgemeinzustand und/oder anderweitig sehr stark erhöhtem Narkoserisiko von einem mikrochirurgischen Eigengewebsaufbau abgesehen werden sollte.

Nach umfangreichen retrospektiven Studien ist weder die Adipositas noch das Alter der Patientin oder eine vorausgegangene Bestrahlung ein signifikanter Risikofaktor für die Lappentransplantation an sich, sodass bei grundsätzlich vertretbarem Narkoserisiko ein freier mikrochirurgischer Eigengewebsaufbau auch in diesen genannten Fällen uneingeschränkt durchgeführt werden kann. 


\section{Spezifische Kontraindikation}

Thrombophilien. Eine spezifische Kontraindikation für jede Form der freien mikrochirurgischen Lappenplastik stellen hingegen ausgeprägte Gerinnungsstörungen im Sinne einer Thrombophilie dar. Hierzu gehören:

- APC-Resistenz (in der Regel auf der Faktor-V-LeidenMutation basierend)

- Prothrombinmutation G20210A (Faktor II)

- Protein-C- und Protein-S-Mangel

- Antithrombin-Mangel

- Antiphospholipid-Antikörper

Diese o.g. Fälle sollten durch ausführliche Anamnese (einschließlich Familienanamnese) bez. thromboembolischer Ereignisse in der Vorgeschichte sowie routinemäßiger Gerinnungsdiagnostik im Vorfeld der operativen Therapie nach Möglichkeit identifiziert werden.

Notwendigkeit von Alternativverfahren. Bei nachgewiesen signifikant erhöhtem Risiko thromboembolischer Komplikationen sollte von einer mikrochirurgischen Lappenplastik abgesehen und auf ein alternatives Verfahren ausgewichen werden. Hierzu gehören, sollte keine vorausgegangene Bestrahlung erfolgt sein und der Haut-/Weichteilmantel suffizient sein, die Expander-/Prothesenimplantation.

In allen Fällen mit vorausgegangener Bestrahlung und/ oder unzureichendem Hautweichteilmantel stellt der gestielte Latissimus-dorsi-Lappen das Ausweichverfahren der Wahl dar, wobei hier zumeist eine zusätzliche Silikongel-Prothesenimplantation zur Erzielung eines ausreichenden Rekonstruktionsvolumens erforderlich wird.

Eine gestielte TRAM-Lappenplastik käme hierfür potenziell ebenfalls infrage, jedoch ist diese aus Hebemorbiditätssicht (Entfernung eines ganzen Rectusabdominis-Muskels) zumindest der Latissimus-Lappenplastik nicht überlegen, hinsichtlich ihrer Sicherheit in der Durchblutung aber unterlegen (s. hierzu auch den Abschnitt TRAM/DIEP-Lappen).

Alternativverfahren sind die Expander- oder Prothesenimplantation sowie gestielte Lappenplastiken.

Hämophilien. Auch ausgeprägte Blutungsneigungen (Hämophilien), die vergleichsweise seltener vorkommen, stellen eine mögliche Kontraindikation für jeden größeren Eingriff dar, d.h. sowohl gefäßgestielte als auch freie Lappenplastiken, sodass in diesen Fällen die Indikationsstellung daher sehr differenziert abgewogen werden sollte.

\section{Freie muskelsparende ventrale Verfahren (TRAM, DIEP, SIEA)}

\author{
Freier transverser Rectus-abdominis- \\ myokutan-Lappen (TRAM) und DIEP-Lappen
}

Der freie muskelsparende transverse Rectus-abdominis-myokutan-Lappen (TRAM) und der DIEP-Lappen („Deep inferior epigastric Perforator“) sind die am häufigsten zum Einsatz kommenden Verfahren in der Eigengewebsrekonstruktion der Brust. Das Gewebe wird hierfür aus der Unterbauchregion entnommen, wobei prinzipiell das Areal zwischen Nabel (kranial) und Symphyse (kaudal) sicher durch das zugehörige Stielgefäß der A. und V. epigastrica inferior durchblutet wird.

Komplikationsrate. Im Rahmen der Weiterentwicklung vom gestielten TRAM-Lappen, welcher von der A. und V. epigastrica superior versorgt wird, konnte gezeigt werden, dass die Verwendung des inferioren Gefäßstiels (freier TRAM-Lappen) mit einer signifikant niedrigeren Komplikationsrate einhergeht, insbesondere hinsichtlich Lappenspitzennekrosen, als bei Verwendung des superioren Gefäßstiels (kranial gestielter TRAM-Lappen) [8]. Dies ist anhand der anatomischen Verhältnisse mit einer signifikant höheren Flussrate in den inferioren vs. superioren epigastrischen Gefäßen naheliegend und konnte sowohl in mehreren experimentellen als auch klinischen Studien belegt werden $[9,10]$.

Bei Verwendung des inferioren Gefäßstiels treten deutlich weniger Komplikationen auf als bei superioren Gefäßstielen.

Kosmetisches Ergebnis. Ein zusätzlicher Vorteil in der Weiterentwicklung vom kranial gestielten zum freien TRAM-Lappen ist die mit dem freien Transplantat verbundene Möglichkeit zur flexibleren Platzierung des Transplantats in seiner Orientierung. Hierdurch ergeben sich deutlich mehr Möglichkeiten in der optimalen Transplantateinpassung und Formung der rekonstruierten Brust.

Hebemorbidität. Nicht zuletzt hat aber die Hebemorbidität sowohl des gestielten als auch des freien TRAM-Lappens, die aufgrund der Opferung eines ganzen Rectus-abdominis-Muskels mit einer folglich signifikanten Schwächung der Bauchdecke und daraus u.U. resultierendem „Bulging“ einhergeht, zur Weiter- 
entwicklung des muskelsparenden (ms-) TRAM-Lappens sowie des DIEP-Lappens geführt. Die beiden Letzteren zeichnen sich durch eine Reduktion des mitzuhebenden Muskelanteils auf eine kleine Muskelinsel (ms-TRAM) bzw. auf die vollständige Präservation des Muskels (DIEP) aus. In zahlreichen Studien konnte belegt werden, dass hierdurch die abdominelle Hebemorbidität signifikant gesenkt werden konnte, ohne dass die Sicherheit hinsichtlich des Transplantats selbst sank [11-13].

Die abdominelle Hebemorbidität ist bei der msTRAM- und DIEP-Lappenplastik deutlich reduziert.

Ob entweder ein ms-TRAM- oder ein DIEP-Lappen bei der jeweiligen Patientin durchgeführt werden kann, ist abhängig von der Anzahl, dem Kaliber und der Lokalisation der das subkutane Fettgewebe und die Haut versorgenden Perforatoren aus den inferioren epigastrischen Gefäßen.

Prä- und intraoperative Messverfahren. Neben der intraoperativen Evaluation sowie der direkt präoperativen Ortung mittels Handdoppler-Gerät oder Duplexsonografie hat sich in den vergangenen Jahren in den meisten Zentren die präoperative Durchführung einer Computertomografie-Angiografie (CTA) als Standard durchgesetzt, da hiermit eine sichere präoperative Lokalisationsdiagnostik der Perforatoren erfolgen kann. Sowohl eine weiterhin erhöhte Sicherheit der Trans-

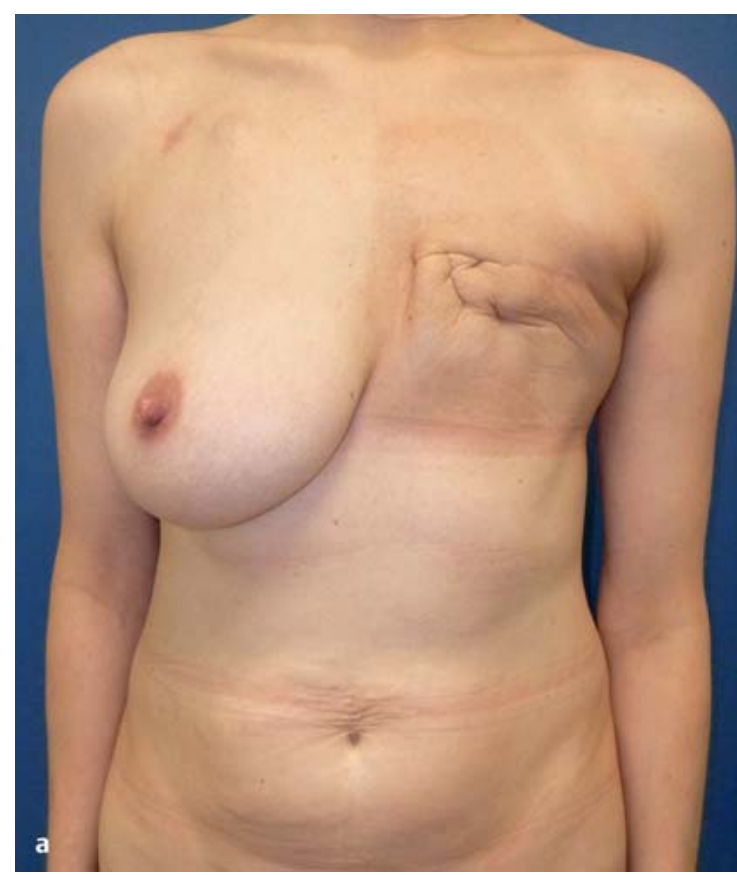

plantation als auch insbesondere eine signifikante Verkürzung der OP-Zeit konnten durch die standardmäßige Einführung der CTA erzielt werden $[14,15]$.

\section{Die CTA hat sich präoperativ zur Lokalisations- diagnostik als Standard etabliert.}

Eine weitere Erhöhung der Sicherheit des Verfahrens sind z.B. intraoperative Messungen der kapillären Sauerstoffsättigung, des postkapillären Hämoglobingehaltes und des Flusses in den verschiedenen Transplantatarealen. Diese Verfahren haben jedoch noch keinen Einzug in die Routine gefunden und kommen v.a. im Rahmen von Studien zur Perfusion des Unterbauchgewebes zum Einsatz [16].

Gewebeverfügbarkeit. Voraussetzung für die Möglichkeit einer ms-TRAM- oder DIEP-Lappentransplantation stellt neben intakten Gefäßverhältnissen der Bauchdecke natürlich eine ausreichende Gewebeverfügbarkeit dar. Es zeigt sich jedoch in der Praxis, dass dies bei der weit überwiegenden Mehrzahl der Patientinnen insbesondere in Korrelation zu der angestrebten Größe der rekonstruierten Brust der Fall ist (Abb. 1 a, b).

Narben, die typischerweise am Rand der Lappenumschneidungsfigur liegen, also v.a. die häufig anzutreffende Appendektomie- und Pfannenstiel-Narbe, stellen keine Kontraindikationen dar, in der Regel ebenso wenig vorausgegangene laparoskopische

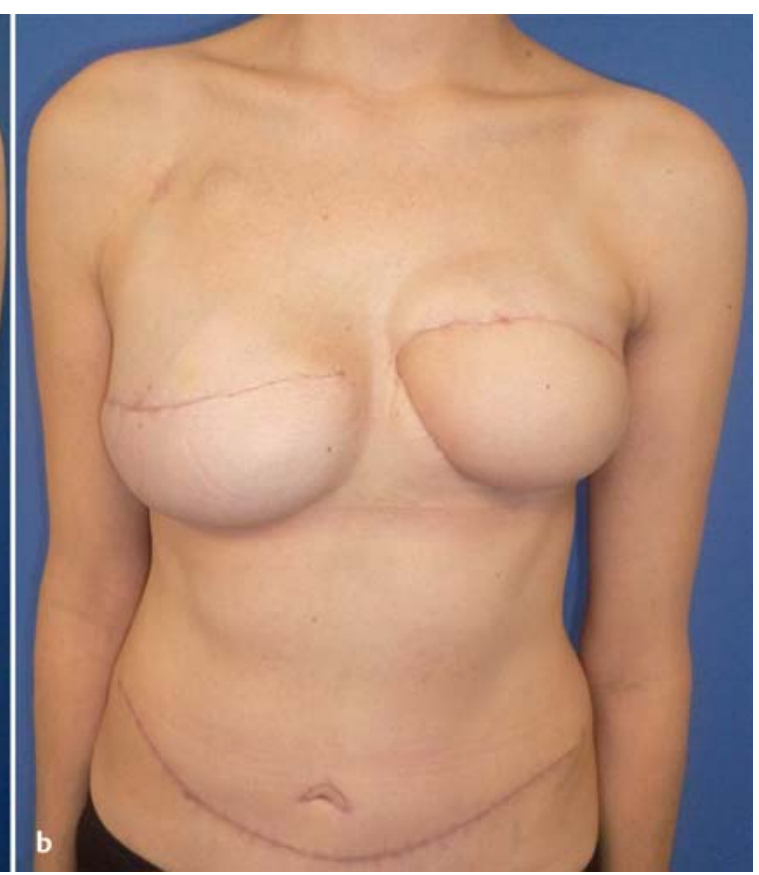

Abb. 1 a Schlanke Patientin (33 J. alt, BMI = 20; 2 Kinder) mit Z.n. Ablatio mammae links. b Z. n. Eigengewebsrekonstruktion der linken Brust mit freiem DIEP-Lappen (Z.n. auswärtiger prophylaktischer Mastektomie und Prothesenimplantation rechte Brust). 
Eingriffe. Bei transumbilikalen Zugängen ist jedoch auf das erhöhte Risiko einer Minderdurchblutung des Nabels nach erfolgter TRAM-Lappenhebung mit evtl. erforderlicher primärer Resektion des Nabels hinzuweisen.

Vorausgegangene abdominelle Narben stellen i. d. R. keine Kontraindikation zur Lappenentnahme dar.

Eine Besonderheit stellt die mediane Laparotomienarbe dar: Grundsätzlich kann hier zwar eine mittellinienübergreifende Perfusion des einen Gefäßstiels vorliegen, die eine normale ms-TRAM/DIEP-Lappentransplantation ermöglicht [17], jedoch sollte im Einzelfall abgewogen werden, ob die Verpflanzung des einen hälftigen Unterbauchfettgewebes (sog. „hemi-msTRAM, -DIEP“) eine ausreichende Gewebemenge darstellt, oder ggf. ein anderes Spenderareal vorzuziehen ist.

\section{SIEA-Lappenplastik}

Eine noch relativ neue Weiterentwicklung des DIEPLappens ist die SIEA-Lappenplastik, bei welcher die im subkutanen abdominellen Fettgewebe verlaufende superfizielle inferiore epigastrische Gefäßachse (SIEA) das Stielgefäß des Lappens darstellt. Diese Lappenplastik zeichnet sich aufgrund ihrer komplett epifaszial gelegenen Hebestelle durch eine minimale Hebemorbidität aus. Allerdings ist zum einen die Gefäßachse der SIEA nicht konstant vorhanden, zum anderen ist das durch sie versorgte Gewebevolumen relativ klein und auf die ipsilaterale Seite der Bauchwand begrenzt [18]. Aus diesem Grund hat sich der SIEA-Lappen in der Breite bis heute nicht durchgesetzt und stellt lediglich eine Ausnahmevariante dar für Patientinnen mit geplantem DIEP-Lappen und intraoperativ sehr stark ausgeprägter SIEA, die nur eine kleinvolumige Brustrekonstruktion benötigen.

\section{Fazit für die Praxis}

Der freie mikrochirurgisch transplantierte ms-TRAModer DIEP-Lappen stellt bei ausreichender Gewebeverfügbarkeit das Verfahren der Wahl zum Eigengewebsaufbau dar, da es mit einer geringeren Hebemorbidität und Lappendurchblutungskomplikationsrate einhergeht als u. a. der kranial gestielte TRAM-Lappen.

\section{Praktische Durchführung}

\section{Einseitige Brustrekonstruktion}

Darstellung der Anschlussgefäße. Die Durchführung eines ms-TRAM/DIEP-Lappens beginnt zunächst mit der Umschneidung der Hautfigur am Unterbauch. Parallel erfolgt durch ein zweites Team die Darstellung der Anschlussgefäße. Hierfür werden in der Regel die A. und V. mammaria interna verwendet, wofür eine kurzstreckige Teilresektion der 3. oder 4. Rippe erforderlich ist (was meist mit keiner von der Patientin spürbaren Morbidität einhergeht; vereinzelt wird auch bei ausreichend weitem Interkostalraum auf die Resektion verzichtet). Alternativ kann die thorakodorsale Gefäßachse als Anschlussgefäß gewählt werden (wobei in diesem Fall sowohl die Strecke zum Transplantat etwas länger ist, als auch die Rückzugsoption eines gestielten Latissimus-dorsi-Transfers nicht mehr zur Verfügung steht).

Präparation des Lappens. Die parallel erfolgende Hebung des Lappens wird zunächst von lateral kommend epifaszial vorgenommen.

Die Präparation sollte unter Lupenbrillenvergrößerung (2- bis 3,5-fache Vergrößerung) durchgeführt werden.

Erst mittelliniennah, d. h. nach Erreichen der ersten mit einzuschließenden Perforatoren, wird das vordere Blatt der Rektusscheide inzidiert und von hier an die Präparation subfaszial unter ggf. Mitnahme eines kleinen Muskelanteils (ms-TRAM) bzw. ausschließlicher Mitnahme des/der versorgenden Perforators/en (DIEP) fortgeführt, bis die inferiore epigastrische Gefäßachse erreicht und ebenfalls bis zu ihrem Abgang aus den externen Iliakalgefäßen disseziert werden kann (Abb. 2a). Hiernach kann der Lappen am Gefäßstiel abgesetzt und in den Empfängersitus verbracht werden.

Gefäßanastomosierung. Im Empfängersitus erfolgt die Anastomosierung der Arterie mit mikrochirurgischer Naht sowie der Vene (ggf. 2 Venen, je nach Verfügbarkeit lappenstiel- und anschlussgefäßseitig) durch entweder ebenfalls Naht- oder Koppleranastomose (Abb. 2 b, c). Mittels einer zusätzlichen Einbettung der anastomosennahen Stielregion mit Fibrinkleber kann eine zusätzliche Sicherheit bei der Vermeidung einer Gefäßstielabknickung („Kinking“) erzielt werden (Abb. 2d). Anschließend wird der Entnahmedefekt analog einer Abdominoplastik mit Nabeltransposition unter Einlage mehrerer Redon-Sogdrainagen ver- 

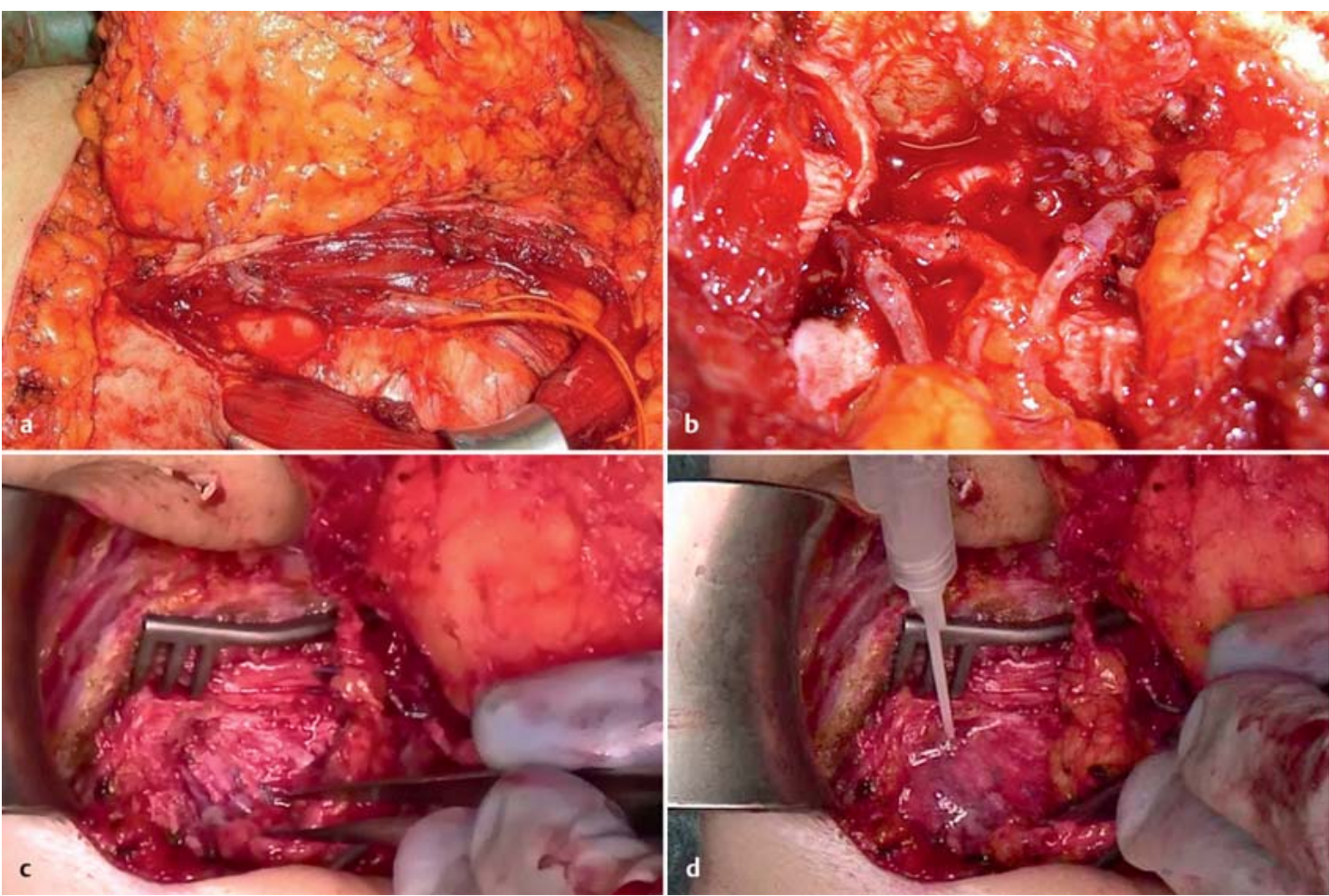

Abb. 2 a Intraoperativer Situs bei Hebung eines DIEP-Lappens mit Darstellung des Perforators und der nach kaudal weiter verlaufenden Gefäßachse der inferioren epigastrischen Gefäße. $\mathbf{b}$ Intraoperativer Situs der Anastomosenregion mit einer arteriellen und 2 venösen (Handnaht-) Anastomosen. c Intraoperativer Situs der Anastomosenregion mit einer arteriellen und einer venösen Koppler-Anastomose. $\mathbf{d}$ Intraoperativer Situs der Anastomosenregion nach Einbettung des anastomosen-nahen Gefäßstiels mit Fibrinkleber.
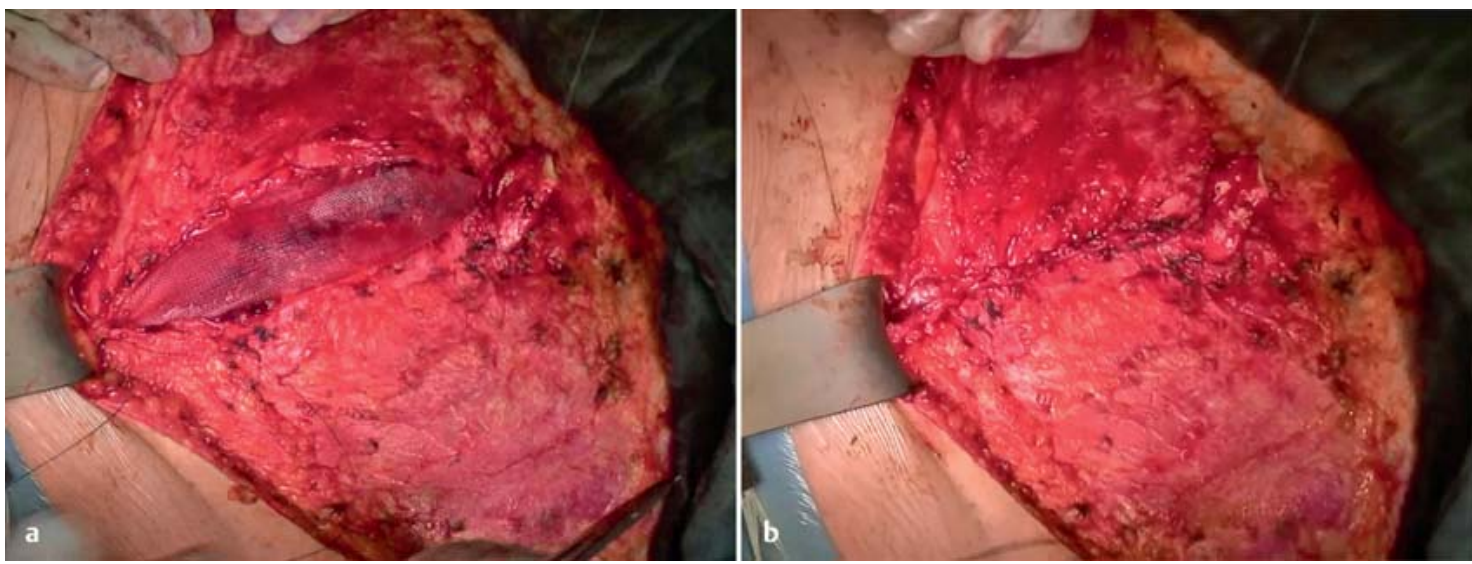

Abb. 3 a Entnahmedefektverschluss durch Abdominoplastik mit Nabeltransposition und Faszienverstärkung durch Implantation eines resorbierbaren Inlay-Netzes (Bauchnabel = rechter Bildrand/Symphyse = linker Bildrand). b Z. n. anatomischer Faszienrekonstruktion über dem darunter liegenden Netz.

schlossen. In der Praxis hat sich hierbei die Implantation eines teilresorbierbaren Netzes als Faszienverstärkung (Abb. 3a) im Sinne einer Inlay-Implantation bewährt, wobei eine anatomische Faszienrekonstruktion (Abb. 3b) in der Regel immer angestrebt werden sollte und bei entsprechend kleinem Fasziendefekt auch immer möglich ist.
Tipp für die Praxis

Die Implantation eines teilresorbierbaren Netzes dient als Faszienverstärkung. Dadurch kann sowohl die Ausbildung von Hernien als auch des ohne Netzimplantation gelegentlich beobachteten „Bulgings“ der Bauchdecke zuverlässig vermieden werden. 
Lappenformung. Im Rahmen der Formung der Brust aus dem ms-TRAM-/DIEP-Lappen werden in der Regel Teile des Lappens deepithelisiert und kranial unter die noch vorhandene Brusthaut untergeschoben, der kaudale Lappenanteil wird entweder ebenfalls komplett oder partiell deepithelisiert und unter die ortsständige Haut, so vorhanden und intakt, untergeschoben oder in der Linie der Unterbrustfalte angeheftet, sodass der Lappen die gesamte ästhetische Einheit der rekonstruierten Brust darstellt (Abb. 4a-d). Durch dieses Vorgehen können in der Regel, abgesehen vom vollständig verborgenen Lappen („Buried Flap“), die kosmetisch besten Ergebnisse erzielt werden (Abb. 5a, b).

\section{Eine kraniale Unterschiebung des teildeepitheli- sierten Lappens gewährleistet optimale kosme- tische Ergebnisse.}

\section{Beidseitige Brustrekonstruktion}

Auch die beidseitige Brustrekonstruktion durch bilaterale ms-TRAM/DIEP-Lappen ist gut möglich. Voraussetzung hierfür ist eine ausreichende Gewebemenge abdominell, um aus jeweils einem Hemi-ms-TRAM/ DIEP-Lappen jeweils eine ausreichend große Brust zu formen. Da es sich hierbei um einen Eingriff höherer Komplexität mit 2 freien mikrochirurgischen Lappentransplantationen in einer Sitzung handelt, muss in Übereinstimmung mit der Datenlage von einer erhöhten intraoperativen und postoperativen Komplikationsrate ausgegangen werden [19]. Als Alternative hierzu ist daher die zweizeitige Rekonstruktion mit jeweils einer freien transversen myokutanen GracilisLappenplastik (TMG, s.u.) pro Sitzung in Erwägung zu ziehen, insbesondere falls die Hebestellen auf den
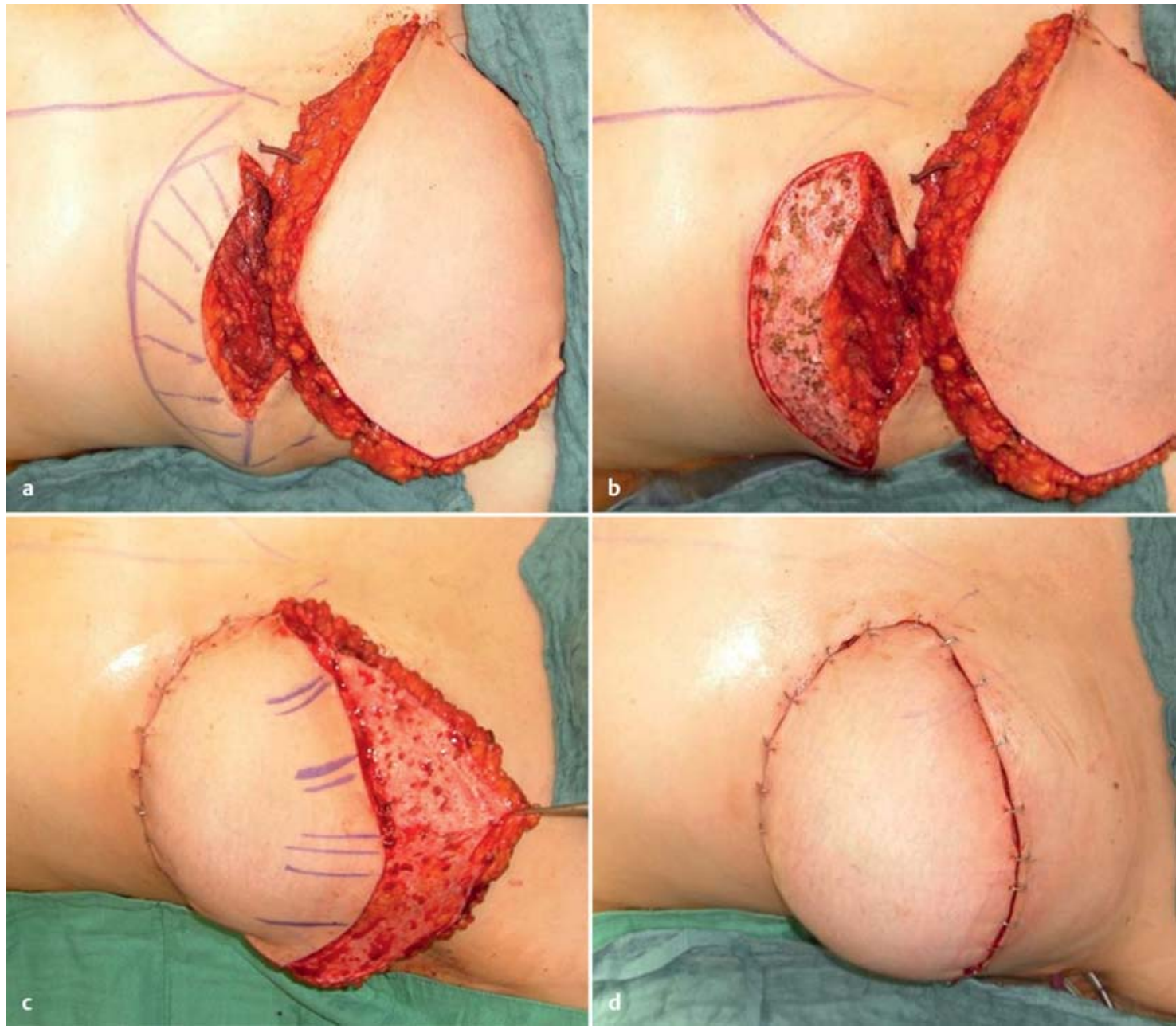

Abb. 4 a Formung der Brust aus dem bereits anastomosierten DIEP-Lappen mit zunächst Anzeichnung des kranial der prospektiven Unterbrustfalte gelegenen und zu deepithelisierenden ortsständigen Hautareals. b Nach Deepithelisierung der ortsständigen Haut. c Nach Anheftung des DIEP-Lappens im Verlauf der Unterbrustfalte und Deepithelisierung der kranial unterzuschiebenden Hautareale des DIEP-Lappens. d Nach Abschluss der Brustformung durch kraniale Unterschiebung der Lappenplastik unter den ortsständigen Hautmantel (probatorischer Hautverschluss mit Hautklammernaht, die zum Abschluss der OP durch eine resorbierbare, fortlaufende Intrakutannaht ersetzt wird). 

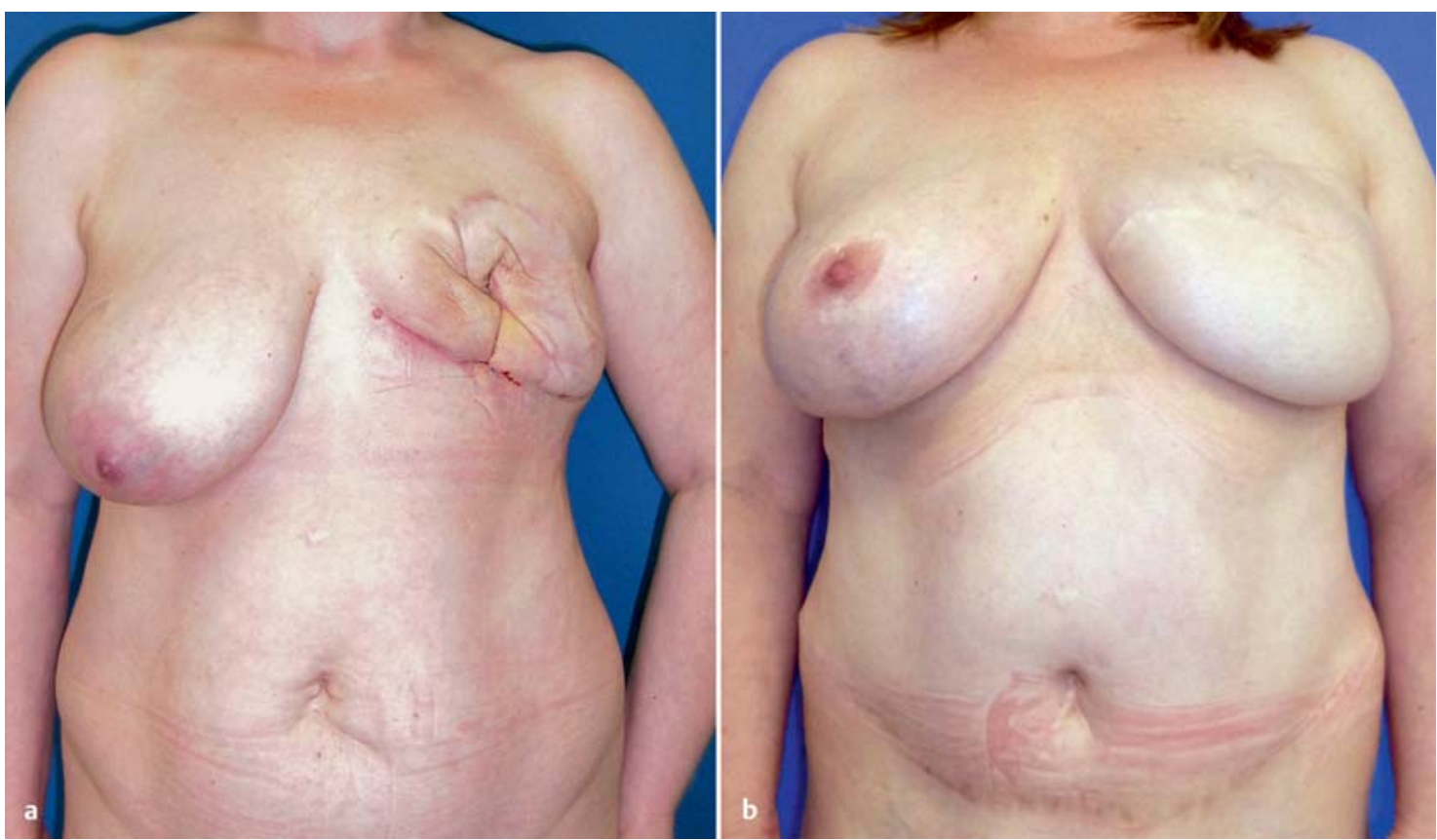

Abb.5 a Präoperativer Befund bei Z.n. Ablatio mammae links bei DCIS, mehrfachen Voroperationen und Protheseninfekten. b Postoperativer Befund nach ms-TRAM-Lappenplastik mit Anheftung des Lappens in der linken Unterbrustfalte und angleichender Mammareduktionsplastik rechts (inv. T-Schnitt-Technik) im Intervall nach 1 Jahr.

Oberschenkelinnenseiten in der Summe eine größere Gewebeverfügbarkeit bieten als die Unterbauchregion.

\section{Nachbehandlung}

Im Rahmen der Nachbehandlung wird neben regelmäßigen, anfangs engmaschigen Durchblutungskontrollen des transplantierten Lappens, die anhand des Rekapillarisierungsmusters des Hautanteils des Lappens vorgenommen werden, häufig eine Bauchbinde oder Kompressionsmiederhose für einen Zeitraum von 6-8 Wochen postoperativ empfohlen. In dieser Zeit sollte auch das Heben schwerer Lasten, d. h. > 5 kg vermieden werden.

Aufgrund einer Reihe von Studien zum Themenkomplex Antikoagulation bei freien Lappenplastiken besteht heutzutage weitestgehend Konsens darüber, dass für den freien ms-TRAM/DIEP-Lappen keine Grundlage für eine antikoagulatorische Therapie besteht, sodass lediglich eine Thromboseprophylaxe mittels gewichtsadaptierter Gabe von fraktioniertem Heparin standardmäßig verabreicht wird.
Weitere spezifische Nachbehandlungsmaßnahmen sind nicht erforderlich. Eine Nachsorge durch z.B. Mammografie oder Magnetresonanztomografie kann problemlos spätestens 6 Monate postoperativ vorgenommen werden.

\section{Fazit freier ms-TRAM/}

DIEP-Lappen

Die Sicherheit des Verfahrens und eine heute deutlich reduzierte OP-Dauer machen den ms-TRAM/DIEPLappen bei ausreichender Gewebeverfügbarkeit zur bevorzugten Methode des Eigengewebsaufbaus. Die routinemäßige Durchführung einer präoperativen Computertomografie-Angiografie hat sich in der Praxis bewährt, erhöht die Sicherheit und verringert die Morbidität des Verfahrens. Eine entsprechende mikrochirurgische Expertise vorausgesetzt, ist der freie ms-TRAM/DIEP-Lappen dem kranial gestielten TRAMLappen sowohl hinsichtlich Lappenkomplikationsrate als auch Hebemorbidität überlegen und sollte soweit verfügbar bevorzugt eingesetzt werden. 


\section{Freier transverser myokutaner Gracilis-Lappen (TMG)}

Als Alternative zum ms-TRAM/DIEP-Lappen hat sich der freie transverse myokutane Gracilis (TMG)-Lappen als Verfahren der ersten Wahl immer mehr durchgesetzt [20,21]. Dies ist u. a. darauf zurückzuführen, dass hier eine sehr hohe Konstanz der Gefäßversorgung vorliegt: Das Stielgefäß, die A. circumflexa femoris medialis, ist praktisch immer vorhanden. Die TMG-Lappenplastik zeichnet sich daher durch eine sehr hohe Sicherheit aus - auch und insbesondere im Vergleich zum sog. superioren Glutealarterien-Perforatorlappen (S-GAP). Darüber hinaus ist die Hebemorbidität kosmetisch verhältnismäßig günstig, da die verbleibende Narbe in Anlehnung an eine horizontale Oberschenkelstraffung in der natürlichen Hautfalte, welche von der Leiste (ventral) medialseitig umlaufend bis zur dorsalen Mittellinie in der Infraglutealfalte reicht, zu liegen kommt [22].

Aufgrund des zumeist geringeren entnehmbaren Hautareals beim TMG-Lappen eignet sich dieses Transplantat v.a. für Patientinnen, bei denen nur ein geringer Teil des Hautmantels zu rekonstruieren ist, d.h.v.a. bei primärer Rekonstruktion nach hautsparender Mastektomie, wie z. B. bei der prophylaktischen Mastektomie oder dem DCIS.

Die entnommene Gewebemenge hinterlässt in Abhängigkeit von der Relation zwischen Haut-/Weichgewebsüberschuss an der Innenseite des Oberschenkels und gehobener Lappendimension u.U. eine asymmetrische Einziehung im Schrittbereich. Diese kann bei Bedarf und Wunsch der Patientin bei einseitiger Entnahme jedoch im Rahmen nachfolgender Korrektureingriffe zumeist problemlos mittels Liposuktion und/ oder modifizierter horizontaler Oberschenkelstraffung angeglichen werden.

Bei beidseitiger Entnahme entfällt diese Problematik, sodass der TMG-Lappen bei Patientinnen mit verhältnismäßig wenig Gewebeüberschuss abdominell bevorzugt bei der beidseitigen Brustrekonstruktion angewandt wird (Abb. 6a, b). Zudem kann hierbei im Gegensatz zum Doppel-ms-TRAM/DIEP-Lappen eine Aufteilung der rekonstruktiven Maßnahmen auf einen freien Gewebetransfer pro Sitzung vorgenommen und damit einhergehend eine Reduktion des intra- und perioperativen Risikos im Vergleich zum Doppel-msTRAM/DIEP-Lappen vorgenommen werden.

\section{Tipp für die Praxis}

Die funktionelle Hebemorbidität des mit zu entnehmenden Gracilis-Muskels ist in Anbetracht der relativen Muskelkraft dieses kleinsten Adduktors minimal [22], allerdings kann es aufgrund der Lokalisation gelegentlich zu länger anhaltender Serombildung und/oder Lymphsekretion kommen, die in der Regel nach einem entsprechenden Zeitraum der Kompressionstherapie sistiert [23].

\section{Praktische Durchführung}

Präparation des Lappens. Die Hebung des freien TMG-Lappens beginnt zunächst mit der Umschneidung der transversen Hautinsel, welche tags zuvor bei der stehenden Patientin eingezeichnet worden war, um so ein Zuliegenkommen der Narbe in der Leisten-, bzw. nach dorsal hin in der Infraglutealfalte zu gewährleisten. Die anterior der vorderen Lappenbegrenzung verlaufende V. saphena magna sollte hierbei geschont
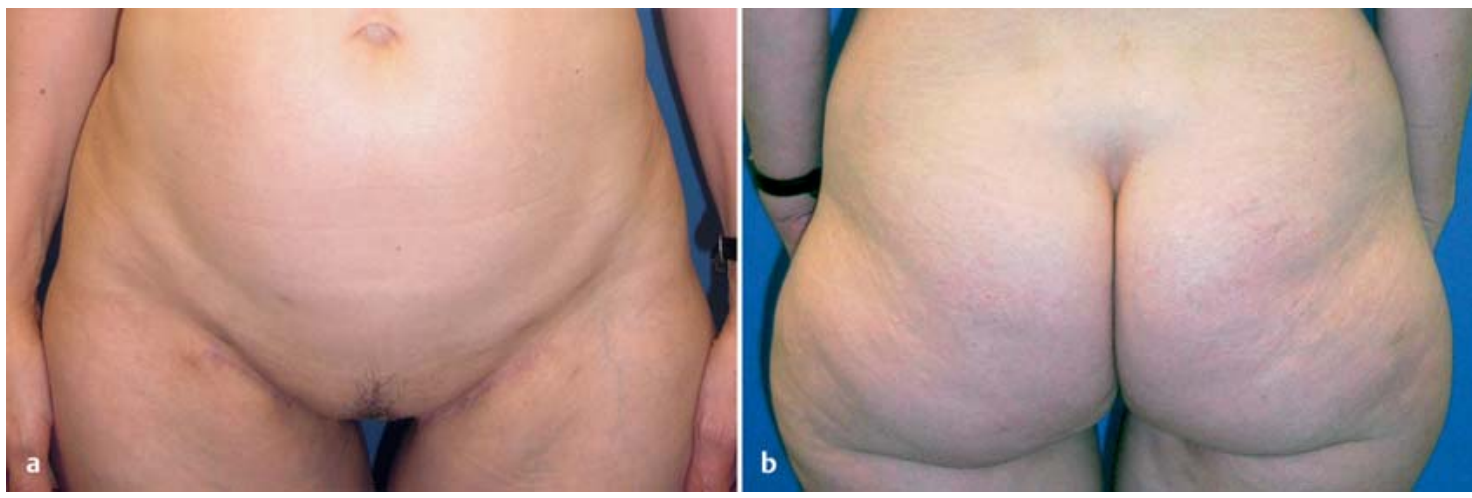

Abb. 6 a Z.n. beidseitiger Entnahme von TMG-Lappen (Frontalansicht mit erkennbaren Ausläufern der Narbe in die Leistenbeugefalte). b Rückenansicht derselben Patientin mit kaum erkennbaren, in den Infraglutealfalten auslaufenden Narben. 

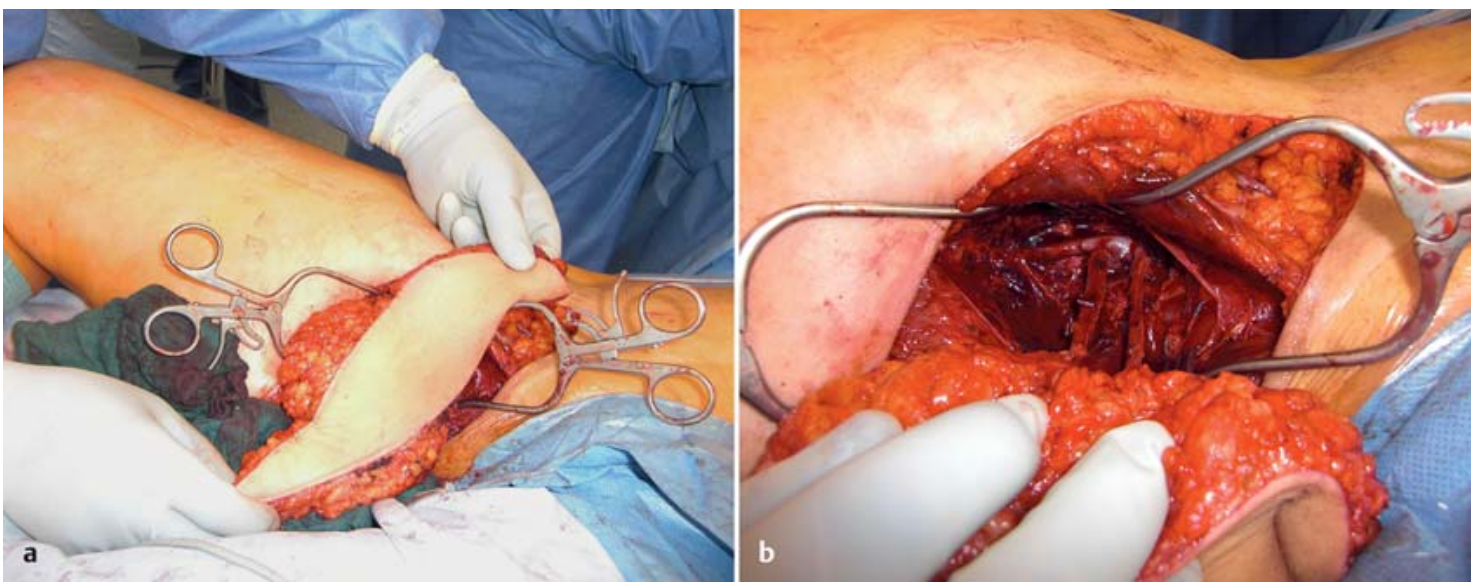

Abb. 7 a Intraoperativer Befund nach vollständiger Mobilisation der transversen Hautinsel des TMG-Lappens (oberer Bildrand = ventral/leistenwärts, unterer Bildrand = nach dorsal/gluteal hin). b Intraoperative Darstellung der Stielgefäße der A./V. circumflexa femoris medialis und ihres Abgangs/ihrer Einmündung in die A./V. profunda femoris (hier mit anatomischer Variante eines sehr kräftigen, ungewöhnlich nah kaudal davon gelegenen sekundären Gefäßstiels, der jedoch nach probatorischem Ausklemmen nicht verwendet werden musste und daher abgesetzt werden konnte).

werden. Nach vollständiger Umschneidung der Hautinsel (Abb. 7a) wird nun der gut palpable M. adductor longus, welcher ventral des M. gracilis liegt, sowie der dorsal hiervon liegende M. adductor magnus zur Darstellung gebracht. Anschließend kann in dem dazwischen liegenden Raum der Gefäßstiel der A. und V. circumflexa femoris medialis bis zu ihrem Abgang bzw. Mündung aus der A. und V. profunda femoris disseziert werden (Abb. 7 b).

Absetzung von Gefäßen und Muskel. Abschließend wird der häufig vorhandene sekundäre Gefäßstiel, welcher weiter distal in den Muskel eintritt, und hiernach der Muskel selbst distal und proximal abgesetzt. Eine separate Inzision im Kniegelenkbereich ist standardmäßig nicht erforderlich. Der mittransplantierte Muskelanteil geht zwar denervierungsbedingt eine mäßige fettige Atrophie durch, jedoch wird die damit einhergehende Volumenreduktion für den Muskelanteil, der ca. 1/3 des Gesamttransplantats ausmacht, mit ca. $20 \%$ angegeben, sodass die Gesamtschrumpfung des Lappens nur sehr gering ist.

Gefäßanastomosierung. Zuletzt werden die Stielgefäße in der Regel mit Durchstechungsligaturen abgesetzt und der Lappen zur Anastomosierung in den Empfängersitus verbracht. Der Lappenanschluss erfolgt dann analog dem ms-TRAM-/DIEP-Lappen und wird unter Mikroskopvergrößerung vorgenommen. Das arterielle Stielgefäß weist hier in der Regel ein kleineres Kaliber als die inferiore epigastrische Arterie auf, die Begleitvene hingegen ist zumeist von gleichem Kaliber wie die V. epigastrica inferior.
Lappenformung. Die Lappenformung wird zunächst durch Fixierung der beiden Enden des Muskelanteils miteinander oder an der ehemals kranialen Begrenzung der Hautinsel vorgenommen, anschließend werden ggf. Teile der Hautinsel deepithelisiert und unter die ortsständige Brusthaut geschoben (Abb. 8a-f).

\section{Nachbehandlung}

Die Nachbehandlung richtet sich auch hier vornehmlich nach der Lappenentnahmestelle, sodass neben dem zumeist empfohlenen 6- bis 8-wöchigen Tragen einer Kompressionsmiederhose lediglich eine Sportkarenz für die ersten postoperativen Wochen eine komplikationslose Abheilung sicherstellen soll.

\section{Fazit freier TMG-Lappen}

Der TMG-Lappen stellt eine sichere Alternative zum ms-TRAM/DIEP-Lappen in Fällen dar, in denen die Gewebeentnahme vom Unterbauch nicht geeignet ist, z. B. aufgrund mangelnder Gewebeverfügbarkeit oder abdomineller Voroperationen. Insbesondere bei Patientinnen mit noch gut erhaltenem Hautmantel der Brust und/oder bei beidseitiger Rekonstruktion stellt der TMG-Lappen eine gute Option zur Brustrekonstruktion dar. Eine präoperative Bildgebung ist aufgrund der großen Konstanz des Gefäßstiels nicht erforderlich. Die Hebemorbidität besteht vornehmlich in der Kosmetik der Oberschenkelinnenseite, die Mitnahme des M. gracilis selbst hinterlässt nur eine minimale funktionelle Hebemorbidität. 

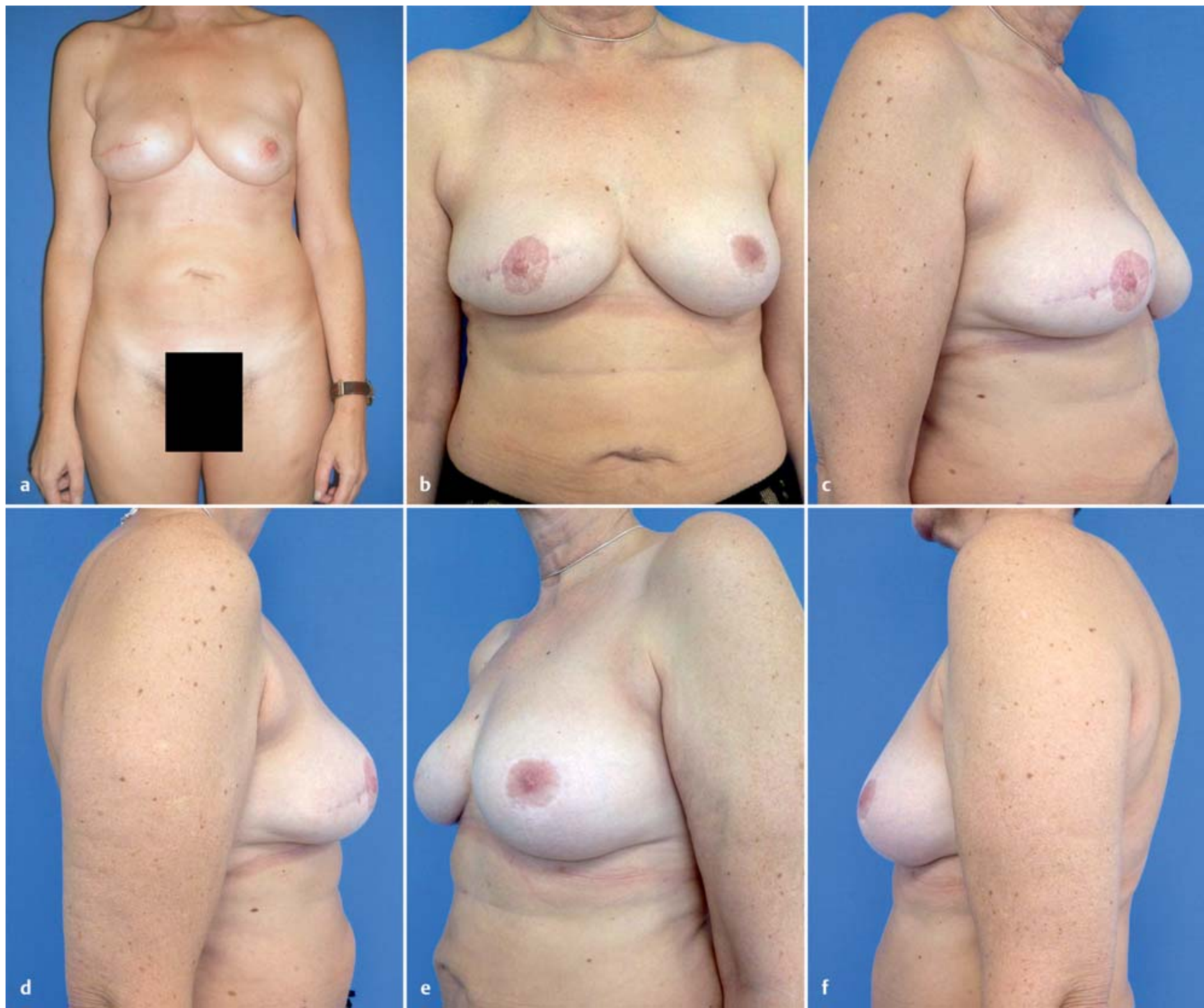

Abb. 8 a Präoperativer Befund bei 39-jähriger Patientin mit Z.n. Ablatio mammae und Expanderimplantation bei multifokalem, invasiv-lobulären Mammakarzinom mit verhältnismäßig geringem Gewebeüberschuss abdominell. b-f Postoperativer Befund 2 Jahre nach autologer Brustrekonstruktion mit freier TMG-Lappenplastik rechts, nachfolgender angleichender Mastopexie links (nach Léjour) und MAK-Rekonstruktion durch Vollhauttransplantation (Entnahme Oberlider bds.) plus Nipple-sharing.

\section{Lappen aus anderen Spenderregionen}

Während der ms-TRAM/DIEP- und der TMG-Lappen in den meisten Zentren die Standardverfahren für die autologe Brustrekonstruktion darstellen, sind der superiore/inferiore Glutealarterien-Perforator (S-/I-GAP)Lappen und der FCI-Lappen eher Ausnahmefällen vorbehalten. Darüber hinaus gibt es jedoch einzelne Einrichtungen, in denen eine spezielle Expertise für eine der o.g. Lappenplastiken vorliegt [24], sodass diese dort weiterhin bevorzugt als 1 . Ausweichalternative zum ms-TRAM/DIEP-Lappen angewandt werden.

\section{Der superiore/inferiore Glutealarterien- Perforator(S-/I-GAP)-Lappen}

S-GAP-Lappen. Beim S-GAP-Lappen, der 1995 erstmalig von R. Allen für die Brustrekonstruktion beschrieben wurde [25], handelt es sich um einen reinen Perforatorlappen. Hier wird analog zum DIEP-Lappen, von der Haut-/Fettgewebsschicht ausgehend, ein geeigneter Perforator in die Tiefe durch den M. glutaeus maximus bis zu dem eigentlichen Stielgefäß, d.h. den superioren Glutealgefäßen in Höhe des Foramen suprapiriforme verfolgt. Anschließend können die Stielgefäße in der Tiefe abgesetzt, durch den in Faserverlaufsrichtung gesplitteten Muskel hindurchgezogen und der Lappen in 
den Empfängersitus transferiert werden. Eine Mitnahme von Glutealmuskulatur selbst erfolgt beim S-GAPLappen nicht.

I-GAP-Lappen. Entsprechend analog erfolgt die Hebung des I-GAP-Lappens, dessen Stielgefäße aus der inferioren Glutealarterie und -vene bestehen und welcher als reiner Perforatorlappen ebenfalls keinen Muskelanteil beinhaltet.

Mit beiden Lappen (S-GAP und I-GAP) ist i. d. R. die Möglichkeit einer relativ großvolumigen Brustrekonstruktion gegeben.

Vorteile. Die Wahl des S-GAP- oder I-GAP-Lappens kann von Vorteil sein im Falle einer bereits einseitig erfolgten Brustrekonstruktion mittels ms-TRAM/DIEPLappen, bei welcher eine nicht geplante Rekonstruktion der Gegenseite im Verlauf erforderlich wird [26], da hier u.U. die ansonsten präferierte TMG-Lappenplastik keine ausreichende Volumenrekonstruktion bieten würde.

Nachteile. In Anbetracht der Entnahmestelle ist jedoch zum einen stets das mitzuhebende Hautareal limitiert, da es hier zu anderenfalls ausgeprägten Wundheilungsstörungen aufgrund der Spannung beim Hebedefektverschluss kommen kann. Zum anderen ist aber auch durch das verbleibende Volumendefizit v.a. beim S-GAP eine sichtbare Einziehung im oberen Gesäßanteil zu befürchten, die kosmetisch nicht zuletzt auch aufgrund ihrer Asymmetrie kritisch zu bewerten ist.

Beim I-GAP besteht hingegen v.a. die Gefahr einer Deformierung des unteren Gesäßanteils mit evtl. damit einhergehender Verstreichung der in diesem Fall kaum wiederherzustellenden Infraglutealfalte. Zudem klagen Patientinnen nach Entnahme eines I-GAP-Lappens zu einem signifikanten Anteil über postoperative Beschwerden beim Sitzen, welche am ehesten zurückzuführen sind auf eine Läsion der in diesem Bereich austretenden $\mathrm{Nn}$. clunei oder des N. cutaneus femoris posterior $[27,28]$.

\section{Der fasziokutane Infragluteal-Lappen (FCl)}

Auch wenn diese genannten Hebemorbiditäten beim FCI-Lappen angeblich geringer sein sollten, stellen auch diese Lappen nicht zuletzt aufgrund des nur in geringem Ausmaß entnehmbaren Hautareals eine Ausnahmeindikation für die Rekonstruktion einer verhältnismäßig kleinen Brust ohne den Bedarf an Hautersatz dar und haben daher bis heute lediglich in wenigen Einrichtungen einen Stellenwert in der routinemäßigen Brustrekonstruktion [29].

\section{Tipp fur die Praxis}

Die freien Lappenplastiken aus der superioren und inferioren Glutealregion sollten aufgrund ihrer Hebemorbidität und der lappenassoziierten Komplikationsraten den wenigen Ausnahmen vorbehalten sein, in welchen weder ms-TRAM/DIEP- noch TMG-Lappen eine Option darstellen.

\section{Sonderfall Latissimus-dorsi-Lappen?}

Der gefäßgestielte Latissimus-dorsi-Lappen hat in der ausschließlich mit Eigengewebe durchgeführten Brustrekonstruktion nur einen sehr geringen Stellenwert, da in aller Regel das damit allein zu erzielende Rekonstruktionsvolumen zu gering ist und eine zusätzliche Silikongelprothesenimplantation erforderlich macht. Aus diesem Grund stellt der alleinige Latissimus-dorsiTransfer eine absolute Ausnahmesituation dar.

Die zusätzliche Verwendung einer Silikongelprothese ermöglicht jedoch eine kosmetisch sehr gute Rekonstruktion einer Brust.

Ein Latissimus-dorsi-Transfer wird meist mit der Transplantation einer Silikongelprothese kombiniert.

Hebemorbidität. Allerdings geht dieses Verfahren mit den Nachteilen beider Prozeduren - wenn auch in unterschiedlicher Gewichtung - einher: Bezüglich der Verwendung des Latissimus-dorsi-Lappens besteht eine signifikante Hebemorbidität durch Entnahme des immerhin größten Muskels des menschlichen Körpers, was bei allen anderen, freien Eigengewebstransplantaten (ms-TRAM/DIEP/SIEA, TMG, S-/I-GAP, FCI) erfolgreich vermieden werden kann. Diese führt neben der sehr häufig zu beobachtenden Serombildung bei vielen Patientinnen zu auch über einen längeren Zeitraum persistierenden Beschwerden in der Schulterfunktion, insbesodere der Elevation und Abduktion des Armes über die Horizontale [30,31].

Auffällige Narben. Zudem ist die zumeist sehr lange Narbe in der Rückenregion zwar, wenn sie längs der Hautspaltlinien in geeigneterer Lokalisation zu liegen kommt, u.U. durch den BH zu verbergen, jedoch insgesamt doch deutlich auffälliger als z.B. die stets durch 
die Unterwäsche zu verbergende ms-TRAM-/DIEP-Entnahme-Narbe oder die in einer natürlichen Hautfalte lokalisierte TMG-Entnahme-Narbe [32].

Prothesenassoziierte Komplikationen. Die in der Regel zusätzlich erforderliche Silikongelprothesenimplantation unter den Muskel hat zwar eine signifikant geringere prothesenassoziierte Komplikationsrate als bei alleiniger Verwendung von Prothesen - insbesondere nach erfolgter Bestrahlung. Allerdings kann es auch hier zu Kapselkontrakturbildung und Prothesendislokation kommen. Die kombinierte Rekonstruktion aus Latissimus-dorsi-Lappen plus Prothese sollte daher nur noch in speziellen Ausnahmefällen durchgeführt werden, bei denen

1. eine freie Lappenplastik kontraindiziert ist (ausgeprägte Thrombophilie, vorausgegangener Verlust eines freien mikrochirurgischen Transplantats, keine vorhandenen Anschlussgefäße) und

2. eine reine Expander-/Prothesenimplantation nicht sinnvoll möglich ist (insuffizienter Haut-/Weichteilmantel, vorausgegangene Bestrahlung).

\section{Kernaussagen}

Für Patientinnen nach karzinombedingter Ablatio mammae oder prophylaktischer Mastektomie stellt sich die Frage nach einer allogenen oder autologen Brustrekonstruktion. Allerdings ist z. B. die Prothesenrekonstruktion der Brust nach einer Strahlentherapie kontraindiziert. Die in den letzten Jahren verbesserte mikrochirurgische Expertise in entsprechenden Zentren führte dazu, dass die autologe Brustrekonstruktion mittels freier mikrochirurgischer Lappenplastiken mittlerweile zum Routineverfahren geworden ist. Neu entwickelte Techniken verwenden unterschiedliche Hebestellen zur Gewinnung von Eigengewebstransplantaten für die Brustrekonstruktion. Die autologe Brustrekonstruktion in freier Technik konnte ihre Überlegenheit gegenüber den früheren Verfahren der gestielten Lappenplastiken sowohl hinsichtlich der kosmetischen Ergebnisse als auch bez. der Komplikationsraten und Sicherheit in zahlreichen Studien belegen. Ungeachtet jeweils vorhandener individueller Einschränkungen kommen prinzipiell folgende freie Verfahren infrage: Bei Lappenentnahmestelle am Unterbauch die TRAM-, DIEP- oder SIEA-Lappentechnik, TMG-Lappen bei Entnahme an der Innenseite des Oberschenkels oder die I-GAP- bzw. S-GAP-Lappen unter Verwendung des Glutealarterien-Perforators sowie der FCI-Lappen. In Kombination mit einer Silikongelprothese kann in Sonderfällen auch ein gefäßgestielter Latissimus-dorsi-Lappen indiziert sein. 


\section{Über die Autoren}

\author{
Justus P. Beier
}

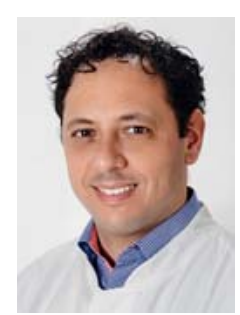

Priv.-Doz. Dr. med. Jahrgang 1976. 1996-2003 Studium der Humanmedizin und Promotion an der Albert-Ludwigs-Universität Freiburg, 2004 Promotion. 2003-2005 Arzt im Praktikum in der Abt. für Plastische und Handchirurgie der Universitätsklinik Freiburg.

Ab 2005 Assistenzarzt in der Plastischund Handchirurgischen Klinik des Universitätsklinikums Erlangen. 2010 Facharztanerkennung Plastische Chirurgie, 2012 Anerkennung der Zusatzbezeichnung Handchirurgie. Seit 2010 Oberarzt, seit 2012 Leitender Oberarzt der Plastisch- und Handchirurgischen Klinik des Universitätsklinikums Erlangen. 2010 Habilitation über das Tissue-Engineering von vaskularisiertem muskoloskelettalem Gewebe. Wissenschaftliche Schwerpunkte: Tissue-Engineering von Skelettmuskel- und Knochengewebe. Klinische Schwerpunkte: Mammachirurgie, rekonstruktive Mikrochirurgie.

\section{Andreas Arkudas}

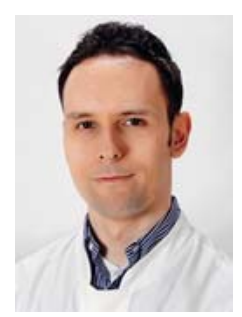

Priv.-Doz. Dr. med. Jahrgang 1977. 1997-2004 Studium der Humanmedizin an der Medizinischen Hochschule Hannover, 2005 Promotion. Ab 2004 Assistenzarzt in der Plastisch- und Handchirurgischen Klinik des Universitätsklinikums Erlangen. 2011 Facharztanerkennung Plastische Chirurgie, 2013 Anerkennung der Zusatzbezeichnung Handchirurgie. Seit 2012 Oberarzt der Plastisch- und Handchirurgischen Klinik des Universitätsklinikums Erlangen. 2012 Habilitation über die Optimierung der Vaskularisation von axial durchbluteten Matrizes im Tissue-Engineering. Wissenschaftliche Schwerpunkte: Tissue-Engineering von Knochengewebe und Gefäßen. Klinische Schwerpunkte: Mammachirurgie, rekonstruktive Mikrochirurgie.

\section{Marweh Schmitz}

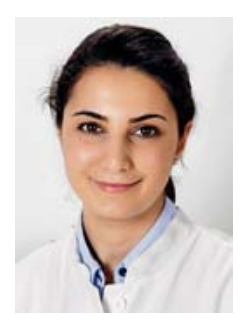

Dr. med. Jahrgang 1979. 1998-2005 Studium der Humanmedizin und Promotion an der Ruhr-Universität Bochum. 2005 Assistenzärztin im Institut für Pathologie der Ruhr-Universität Bochum, BG Unfallklinik Bergmannsheil Bochum. 2005-2008 Assistenzärztin der Klinik für Hand-, Plastische und Re- konstruktive Chirurgie, Schwerbrandverletztenzentrum, BG-Unfallklinik Ludwigshafen. Ab 2008 Assistenzärztin der Plastisch- und Handchirurgischen Klinik des Universitätsklinikums Erlangen. 2012 Facharztanerkennung Plastische Chirurgie. Seit 2014 Oberärztin der Plastisch- und Handchirurgischen Klinik des Universitätsklinikums Erlangen.

Wissenschaftlicher Schwerpunkt: Kapselfibrose bei Silikonprothesenimplantation.

\section{Raymund E. Horch}

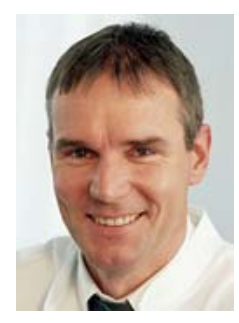

Prof. Dr. med. Jahrgang 1957. 1977 1983 Studium der Medizin in Aachen und Bonn. Promotion an der Rheinischen Friedrich-Wilhelms-Universität Bonn. 1983-1990 Chirurgische Universitätsklinik Bonn, Facharztanerkennung Chirurgie 1990. Wissenschaftlicher Referent MDK 1990-1992, Klinik für Plastische und Handchirurgie, Schwerbrandverletztenzentrum Köln-Merheim, Facharztanerkennung Plastische Chirurgie 1994, Zusatzbezeichnung Handchirurgie 1997. 1994-2002 Stellvertretender Abteilungsleiter Abt. für Plastische und Handchirurgie der Universitätsklinik Freiburg. 1997 Habilitation über Kultivierung von Keratinozyten zum Tissue-Engineering von Hautersatz. 2002 Apl. Professor für Plastische und Handchirurgie Universität Freiburg. Seit 2003 Direktor der Plastisch- und Handchirurgischen Klinik des Universitätsklinikums Erlangen der Friedrich-Alexander-Universität Erlangen-Nürnberg. Seit 2004 Sprecher des Konvents der Leitenden Plastischen Chirurgen der Universitätsklinika in Deutschland. Wissenschaftliche Schwerpunkte: TissueEngineering von Haut, Knochen- und Skelettmuskelgewebe, Mechanismen der Kapselkontraktur und der Wundheilung. Klinische Schwerpunkte: Rekonstruktive Mikrochirurgie, Mammachirurgie, Handchirurgie.

\section{Interessenkonflikt}

Der korrespondierende Autor erklärt für sich und seine Koautoren, dass während der letzten 3 Jahre keine wirtschaftlichen oder persönlichen Verbindungen bestanden, die zu einem Interessenkonflikt mit dieser Publikation führen.

\section{Korrespondenzadresse}

Priv.-Doz. Dr. med. Justus P. Beier

Ltd. Oberarzt, Plastisch- und Handchirurgische Klinik

Universitäts-Brustzentrum Franken

Universitätsklinikum Erlangen

Krankenhausstraße 12

91054 Erlangen

E-Mail: justus.beier@uk-erlangen.de 


\section{Literatur}

$1 \mathrm{Holmstrom} \mathrm{H}$. The free abdominoplasty flap and its use in breast reconstruction. An experimental study and clinical case report. Scand J Plast Reconstr Surg 1979; 13: 423-427

2 Hartrampf CR, Scheflan M, Black PW. Breast reconstruction with a transverse abdominal island flap. Plast Reconstr Surg 1982; 69: 216-225

3 Beier JP, Arkudas A, Horch RE. Is there a paradigm shift in reconstructive breast surgery? GMS Ger Plast Reconstr Aesthet Surg 2013; 3: 1-6

4 Im Internet: http://www.fda.gov/MedicalDevices/ProductsandMedicalProcedures/ImplantsandProsthetics/Breastlmplants/ default.htm. Stand: Mai 2014

5 Langfassung der Leitlinie „Mammakarzinom der Frau: Diagnostik, Therapie und Nachsorge“. Im Internet: http://www.awmf.org/ leitlinien/detail/II/032-045OL.html. Stand: Mai 2014

6 Schaverien MV, Macmillan RD, McCulley SJ. Is immediate autologous breast reconstruction with postoperative radiotherapy good practice? A systematic review of the literature. J Plast Reconstr Aesthetic Surg 2013; 66: 1637-1651

7 McKeown DJ, Hogg FJ, Brown IM et al. The timing of autologous latissimus dorsi breast reconstruction and effect of radiotherapy on outcome. J Plast Reconstr Aesthetic Surg 2009; 62: 488-493

8 Andrades P, Fix RJ, Danilla $S$ et al. Ischemic complications in pedicle, free, and muscle sparing transverse rectus abdominis myocutaneous flaps for breast reconstruction. Ann Plast Surg 2008; 60: $562-567$

9 Hendricks DL, Wilkens TH, Witt PD. Blood-flow contributions by the superior and inferior epigastric arterial systems in TRAM flaps, based on laser Doppler flowmetry. J Reconstr Microsurg 1994; 10: 249-252; discussion 253-244

10 Taylor $\mathrm{Gl}$. The angiosomes of the body and their supply to perforator flaps. Clin Plast Surg 2003; 30: 331-342

11 Wu LC, Bajaj A, Chang DW et al. Comparison of donor-site morbidity of SIEA, DIEP, and muscle-sparing TRAM flaps for breast reconstruction. Plast Reconstr Surg 2008; 122: 702-709

12 Schaverien MV, Perks AG, McCulley SJ. Comparison of outcomes and donor-site morbidity in unilateral free TRAM versus DIEP flap breast reconstruction. J Plast Reconstr Aesthetic Surg 2007; 60: $1219-1224$

13 Bajaj AK, Chevray PM, Chang DW. Comparison of donor-site complications and functional outcomes in free muscle-sparing TRAM flap and free DIEP flap breast reconstruction. Plast Reconstr Surg 2006; 117: 737-746; discussion 747-750

14 Ghattaura A, Henton J, Jallali $\mathrm{N}$ et al. One hundred cases of abdominal-based free flaps in breast reconstruction. The impact of preoperative computed tomographic angiography. J Plast Reconstr Aesthetic Surg 2010; 63: 1597-1601

15 Smit JM, Dimopoulou A, Liss AG et al. Preoperative CT angiography reduces surgery time in perforator flap reconstruction. J Plast Reconstr Aesthetic Surg 2009; 62: 1112-1117

16 Beier JP, Horch RE, Arkudas A et al. Decision-making in DIEP and ms-TRAM flaps: the potential role for a combined laser Doppler spectrophotometry system. J Plast Reconstr Aesthetic Surg 2013; 66: $73-79$
17 Dragu A, Unglaub F, Wolf MB et al. Scars and perforator-based flaps in the abdominal region: a contraindication? Can J Surg 2010; 53: 137-142

18 Munhoz AM, Pellarin L, Montag E et al. Superficial inferior epigastric artery (SIEA) free flap using perforator vessels as a recipient site: clinical implications in autologous breast reconstruction. Am J Surg 2011; 202: 612-617

19 Wormald JC, Wade RG, Figus A. The increased risk of adverse outcomes in bilateral deep inferior epigastric artery perforator flap breast reconstruction compared to unilateral reconstruction: a systematic review and meta-analysis. J Plast Reconstr Aesthetic Surg 2014; 67: 143-156

20 Fansa H, Schirmer S, Warnecke IC et al. The transverse myocutaneous gracilis muscle flap: a fast and reliable method for breast reconstruction. Plast Reconstr Surg 2008; 122: 1326-1333

21 Schoeller T, Huemer GM, Wechselberger G. The transverse musculocutaneous gracilis flap for breast reconstruction: guidelines for flap and patient selection. Plast Reconstr Surg 2008; 122: 29 38

22 Pulzl P, Schoeller T, Kleewein K et al. Donor-site morbidity of the transverse musculocutaneous gracilis flap in autologous breast reconstruction: short-term and long-term results. Plast Reconstr Surg 2011; 128: 233e-242e

23 Buntic RF, Horton KM, Brooks D et al. Transverse upper gracilis flap as an alternative to abdominal tissue breast reconstruction: technique and modifications. Plast Reconstr Surg 2011; 128: 607e-613e

24 Papp C, Windhofer C, Michlits W. Autologous breast augmentation with the deepithelialized fasciocutaneous infragluteal free flap: a 10-year experience. Ann Plast Surg 2011; 66: 587-592

25 Allen RJ, Tucker C jr. Superior gluteal artery perforator free flap for breast reconstruction. Plast Reconstr Surg 1995; 95: 1207-1212

26 Beier JP, Horch RE, Bach AD. Breast reconstruction after breastcancer surgery. N Engl J Med 2009; 360: 418-419; author reply 420-421

27 Godbout E, Farmer L, Bortoluzzi P et al. Donor-site morbidity of the inferior gluteal artery perforator flap for breast reconstruction in teenagers. Can J Plast Surg 2013; 21: 19-22

28 Guerra AB, Metzinger SE, Bidros RS et al. Breast reconstruction with gluteal artery perforator (GAP) flaps: a critical analysis of 142 cases. Ann Plast Surg 2004; 52: 118-125

29 Papp C, Windhofer C, Gruber S. Breast reconstruction with the fasciocutaneous infragluteal free flap (FCI). Ann Plast Surg 2007; 58: $131-136$

30 Clough KB, Louis-Sylvestre C, Fitoussi A et al. Donor site sequelae after autologous breast reconstruction with an extended latissimus dorsi flap. Plast Reconstr Surg 2002; 109: 1904-1911

31 Adams WP jr, Lipschitz AH, Ansari M et al. Functional donor site morbidity following latissimus dorsi muscle flap transfer. Ann Plast Surg 2004; 53: 6-11

32 Kelly MB, Searle A. Improving the donor site cosmesis of the latissimus dorsi flap. Ann Plast Surg 1998; 41: 629-632 


\section{CME•thieme.de}

CME-Teilnahme

- Viel Erfolg bei lhrer CME-Teilnahme unter http://cme.thieme.de

- Diese Fortbildungseinheit ist 12 Monate online für eine CME-Teilnahme verfügbar.

- Sollten Sie Fragen zur Online-Teilnahme haben, unter

http://cme.thieme.de/hilfe finden Sie eine ausführliche Anleitung.

\section{CME-Fragen}

Welches Verfahren zur Brustrekonstruktion sollte nach vorausgegangener Bestrahlung des Mastektomieareals grundsätzlich nicht mehr durchgeführt werden?

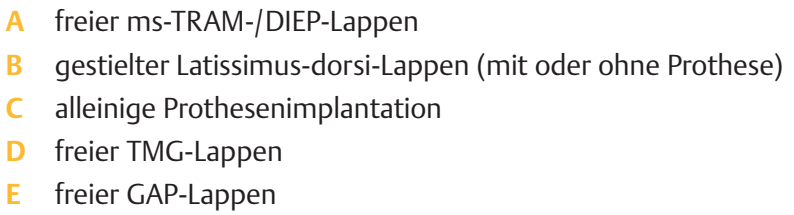

2 Welcher Parameter spielt bei der Indikationsstellung zum Eigengewebsaufbau der Brust keine entscheidende Rolle?

A Wunsch der Patientin

B angestrebtes rekonstruktives Ergebnis

C lokale Gewebeverfügbarkeit an potenziellen Entnahmestellen

D nominelles Alter

E individuelles Risikoprofil der Patientin

3 Welche Aussage ist richtig? Der Eigengewebsaufbau kann ...

A sowohl als primäre als auch als sekundäre Rekonstruktion erfolgen.

B ausschließlich sekundär erfolgen.

C als primäre Rekonstruktion ausschließlich bei invasiven Tumoren durchgeführt werden.

D als sekundäre Rekonstruktion ausschließlich bei In-situ-Tumoren erfolgen.

E bei prophylaktischer Mastektomie nicht primär erfolgen.

4 Für die Eigengewebsrekonstruktion der Brust mit Gewebe vom Unterbauch ist folgende Aussage richtig:

A Der kranial gestielte TRAM-Lappen ist hinsichtlich seiner Lappendurchblutung statistisch und nach Studienlage als sicherer einzuschätzen als der freie Lappen.

B Der freie muskelsparende TRAM-Lappen hat eine größere Hebemorbidität als der kranial gestielte TRAM-Lappen.

C Der freie ms-TRAM-Lappen und der DIEP-Lappen unterscheiden sich nicht signifikant hinsichtlich ihrer Hebemorbidität, aber beide unterscheiden sich wieder vom kranial gestielten TRAM-Lappen durch eine geringere Hebemorbidität.

D Die superiore epigastrische Gefäßachse ist in der Regel deutlich kräftiger ausgeprägt als die inferiore.

E Die Möglichkeiten bei der Formung der zu rekonstruierenden Brust sind deutlich größer bei der gestielten versus der freien TRAM-Lappenplastik.

Welche Aussage ist richtig? Eine Rekonstruktion durch freie mikrochirurgische Lappentransplantation aus der Unterbauchregion ...

A kann bei Vorliegen einer medianen Laparotomienarbe grundsätzlich nicht mehr durchgeführt werden.

B geht bei beidseitiger Rekonstruktion durch Doppel-ms-TRAM/DIEP-Lappen nicht mit einer erhöhten Komplikationsrate im Vergleich zur einseitigen Rekonstruktion einher.

C sollte nicht nach vorausgegangener Bestrahlung erfolgen.

D kann bei ausreichender Gewebeverfügbarkeit zur Rekonstruktion beider Brüste durchgeführt werden.

E kann nur bei noch vorhandener A./V. thoracodorsalis als Anschlussgefäß erfolgen. 
Welche Aussage ist richtig?

A Der funktionelle Hebedefekt des TMG-Lappens ist i. d. R. deutlich höher als der kosmetische Hebedefekt einzustufen.

B Die Hebung des TMG-Lappens erfolgt i.d. R. unter vollständiger Schonung der Adduktorenmuskulatur.

C Die Gefäßversorgung des TMG-Lappens erfolgt über die A. circumflexa femoris lateralis.

D Der TMG-Lappen ist hinsichtlich seiner anatomischen Verhältnisse durch eine große Konstanz insbesondere bez. der Hauptgefäßversorgung gekennzeichnet.

E Mittels freier TMG-Lappenplastik kann in der Regel ein großflächiger Hautmantelersatz durch Eigengewebe, wie er v. a. für die sekundäre Brustrekonstruktion ohne vorherige Expanderimplantation benötigt wird, erzielt werden.

Welche Aussage ist falsch?

A Bei Patientinnen mit schlankem Habitus und keinem ausreichenden Gewebeüberschuss am Bauch ist eine autologe Brustrekonstruktion i.d. R. nur vom Gesäß möglich.

B Bei Patientinnen mit schlankem Habitus und ausreichendem Gewebeüberschuss am Bauch ist i.d. R. die Verwendung eines ms-TRAM/DIEP-Lappens die beste Option zur autologen Brustrekonstruktion.

C Bei Patientinnen mit schlankem Habitus und ausgeprägtem Gewebeüberschuss an den Oberschenkelinnenseiten stellt der TMG-Lappen eine sichere Alternative zum ms-TRAM/DIEP-Lappen dar.

D Bei Patientinnen mit multiplen abdominellen Voroperationen kann die TMG-Lappenplastik eine sichere alternative Option darstellen.

E Bei Patientinnen mit beidseitiger Ablatio mammae ist eine beidseitige Rekonstruktion mit beidseitig zu hebenden TMG-Lappen eine Alternative zum Doppel-ms-TRAM/DIEP-Lappen.

$8 \quad$ Welche Aussage ist falsch?

A Der freie S-GAP-Lappen geht ohne Entnahme von Glutealmuskulatur einher.

B Der Entnahmedefekt beim S-GAP-Lappen stellt v. a. bei schlanken Patientinnen meist keine kosmetische Beeinträchtigung dar.

C Der S-GAP-Lappen ist hinsichtlich der Komplexität seiner Präparation bei der Hebung als technisch schwieriger im Vergleich zum TMG-Lappen einzustufen.

D Bei Patientinnen mit sehr schlankem Habitus und ohne entsprechenden Gewebeüberschuss an Bauch und Oberschenkelinnenseite stellt der S-GAP-Lappen auch zur beidseitigen Rekonstruktion eine mögliche Alternative zur Eigengewebsrekonstruktion dar.

E Der S-GAP-Lappen wird frei an den superioren Glutealgefäßen als Gefäßstiel transplantiert.

\section{Welche Aussage zu I-GAP- und FCI-Lappen ist richtig?}

A Der I-GAP- und FCl-Lappen sind freie mikrochirurgische Lappenplastiken aus der superioren Glutealregion.

B Eine relevante Komplikationsmöglichkeit bei diesen Lappen stellen Sensibilitätsstörungen im Bereich des anterioren Oberschenkels dar.

C Beide Lappenplastiken gehören inzwischen weltweit zum standardmäßigen Repertoire der rekonstruktiven Brustchirurgen. D Beim I-GAP-Lappen wird der inferiore Teil der Glutealmuskulatur mitgehoben.

E Der FCl-Lappen zeichnet sich durch eine verhältnismäßig kleine mitzuhebende Hautinsel aus und ist deshalb vornehmlich für Patientinnen mit prophylaktischer und/oder subkutaner Mastektomie und kleiner Brust geeignet. 
Welche Aussage ist falsch?

A Als gestielter Lappen ist der Latissimus-dorsi-Lappen (plus ggf. Prothesenimplantation) eine wichtige Rückzugsoption, falls es Kontraindikationen gegen freie mikrochirurgische Verfahren (wie z. B. nachgewiesene Gerinnungsstörungen) gibt.

B Der Entnahmedefekt des Latissimus-dorsi-Lappens wird heutzutage sowohl hinsichtlich der Schulterfunktion als auch der Kosmetik als nachteilig gegenüber dem ms-TRAM/DIEP- und dem TMG-Lappen angesehen.

C Bei der Brustrekonstruktion mit gestieltem Latissimus-dorsi-Lappen ist i.d. R. keine zusätzliche Prothesenimplantation erforderlich.

D Ein Latissimus-dorsi-Lappentransfer kann auch nach vorausgegangener (erfolgloser) freier mikrochirurgischer Lappenplastik mit Anschluss an die A./V. mammaria interna noch durchgeführt werden.

E Der Latissimus-dorsi-Lappen sollte aufgrund seiner Hebemorbidität und der Notwendigkeit der zusätzlichen Prothesenimplantation heute nicht mehr das Verfahren der ersten Wahl in der Brustrekonstruktion unter Verwendung von Eigengewebe darstellen. 